\title{
Dopamine is a double-edged sword: Enhancing memory retrieval performance at the expense of metacognition
}

\author{
Mareike $\mathrm{Clos}^{1 *}$, Nico Bunzeck ${ }^{1,2}$, Tobias Sommer ${ }^{1}$ \\ ${ }^{1}$ Department of Systems Neuroscience, University Medical Center Hamburg-Eppendorf, Germany. \\ ${ }^{2}$ Institute of Psychology I, University of Lübeck, Lübeck, Germany. \\ *Correspondence to: m.clos@uke.de
}

\begin{abstract}
While memory encoding and consolidation processes have been linked with dopaminergic signaling for a long time, the role of dopamine in episodic memory retrieval remained mostly unexplored. Based on previous observations of striatal activity during memory retrieval, we used pharmacological fMRI to investigate the effects of dopamine on retrieval performance and metacognitive memory confidence in healthy humans. Dopaminergic modulation by the D2 antagonist haloperidol administered acutely during the retrieval phase improved recognition accuracy of previously learned pictures significantly and was associated with increased activity in the SN/VTA, locus coeruleus, hippocampus and amygdala during retrieval. In contrast, confidence for new-decisions was impaired by unsystematically increased activity of the striatum across confidence levels and restricted range of responsiveness in frontostriatal networks under haloperidol. These findings offer new insights into the mechanisms underlying memory retrieval and metacognition and provide a broader perspective on the presence of memory problems in dopamine-related diseases and the treatment of memory disorders.
\end{abstract}

\section{Keywords}

dopamine, episodic memory retrieval, fMRI, haloperidol, striatum 


\section{Introduction}

Numerous studies have specified the contribution of the medial temporal lobe as well as prefrontal and parietal areas to the retrieval of episodic information. However, besides this established retrieval network also striatal activity is consistently observed during memory retrieval (Kim, 2013), which might point to a hitherto uncharted role of dopaminergic neurotransmission in retrieval processes. In contrast to retrieval, encoding and consolidation of memories are well-known to require the stimulation of dopamine receptors in the hippocampus, striatum, amygdala and prefrontal cortex (PFC) (Mele et al, 2004; Rocchetti et al, 2015; Rossato et al, 2013) as part of a functional loop that orchestrates the formation of new memories (Axmacher et al, 2010; Lisman and Grace, 2005). Striatal dopaminergic influence during retrieval, on the other hand, has been suggested to reflect motivational aspects such as higher subjective value of successfully retrieving old than rejecting new items in a memory test (Han et al, 2010) or higher memory confidence during retrieval (Schwarze et al, 2013). Intriguingly, however, dopamine might also affect the actual retrieval of episodic information, for example it could potentially support cognitive control processes during retrieval (Scimeca and Badre, 2012) or improve the signal-to-noise ratio of memory representations (Frank, 2005; Warren et al, 2016; Yousif et al, 2016). In a previous fMRI study involving a picture recognition task, we provided evidence for independent retrieval-related and memory confidence signals in overlapping striatal regions (Clos et al, 2015). We here investigated directly whether these striatally-mediated memory processes are indeed dopaminergic. In order to disentangle the potential dopaminergic effects on memory confidence and retrieval performance, we designed a randomized, placebo-controlled between-group pharmacological fMRI study. We used the same recognition task as in our previous study (Clos et al, 2015), in which the participants had to rate the old/new status of pictures and their memory confidence on a combined 6-point confidence scale. Dopamine level was manipulated during retrieval by $2 \mathrm{mg}$ of the D2-antagonist haloperidol administered only after encoding. 


\section{Materials and Methods}

\section{Participants and group allocation}

Fifty-four volunteers ( 14 males, mean age $24 \pm 2.9$ years) participated in the study. We used a doubleblind, placebo-controlled between-group design, assigning 27 participants randomly to either placebo or haloperidol group. The sample size was based on our experience in earlier fMRI studies using the same picture recognition paradigm (Clos et al, 2015; Schwarze et al, 2013) and previous pharmacological fMRI studies comparing haloperidol and placebo using between-group designs (Cole et al, 2013; Menon et al, 2007; Oei et al, 2012; Pessiglione et al, 2006; Pleger et al, 2009; Wrobel et al, 2014). We chose for a between-group design rather than for the more powerful within-group crossover design to avoid interference between the highly similar picture stimuli used in the recognition paradigm and because of additional tasks measured in this sample (Clos et al, in revision), which were incompatible with a within-group design. The random group assignment was conducted by TS, who did not interact with the participants at any time. All other experimenters were blind with regard to group assignment and group allocation was only revealed after the measurement of the last participant was completed. The study was approved by the local ethics committee of the Hamburg medical association and written consent was given by each participant prior to the start of the study. Additionally, all participants were screened by a physician for previous or current physical or mental diseases, medication or drug use, ensuring that only healthy participants were included in the study. The participants were informed about the purpose and the course of the study and about the potential risks and side effects of haloperidol. Moreover, the participants were instructed to restrain from caffeine, nicotine, and alcohol on the day of the fMRI testing. Repeated blood pressure and pulse measurements together with questionnaires on adverse medication effects and current mood ensured the well-being of the participants after haloperidol/placebo administration. All participants were asked to indicate the substance (haloperidol or placebo) they thought they had received and how certain they were of this guess at the end of the study. 


\section{Experimental design}

Behavioral working-memory baseline and encoding session (TO)

Encoding for the fMRI experiment took place outside the scanner on the first day within a one-hour baseline testing session (no medication given). The participants first completed working memory (WM) baseline tests on a computer. We measured their performance on a self-paced complex span task (Unsworth et al, 2009), where the location of 2-5 squares within a 16-square pattern had to be remembered while simultaneously judging the symmetry of abstract pictures (symmetric/asymmetric). Subsequently, the participants performed a self-paced digit and block span task (Kessels et al, 2008). Digit sequences presented auditorily via headphones had to be entered via the keyboard after presentation of the last digit in either forward or backward order. For the forward digit span, the sequence increased from 3 digits to maximally 8 digits (depending on performance). For the backward digit span, the sequence increased from 2 digit to maximally 7 digits. Similarly, during trials of the block span task, the participants saw a pattern of objects, whose location and (forward/backward) appearance order they had subsequently to indicate via mouse clicks on these objects. For the forward block span, the sequence increased from 2 objects to maximally 8 objects. For the backward block span, the sequence increased from 2 to maximally 7 objects.

For the recognition memory task, we used the same encoding paradigm as previously (Clos et al, 2015). In total 80 unfamiliar photos of outdoor scenes (Peelen et al, 2009) were presented for $800 \mathrm{~ms}$. The participants had to indicate the category for each picture (i.e. whether it contained cars or people) by pressing one of two buttons on the keyboard (Fig. $1 \mathrm{~A}$ ). Each picture was followed by an active baseline condition (ISI 8-12 s, arrow pointing task) in which the direction of arrows presented for 800 ms had to be indicated by button press. 

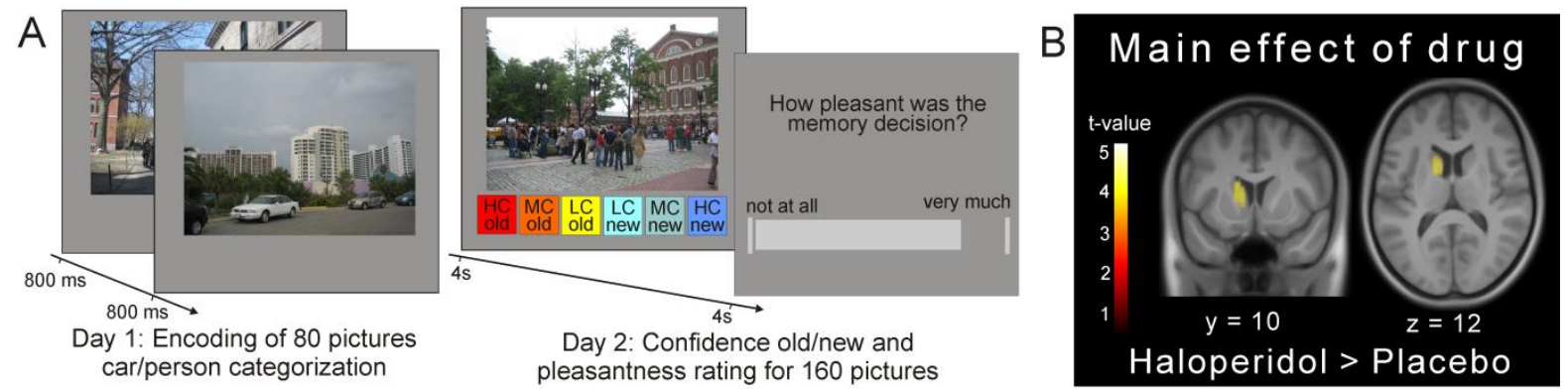

Figure 1: Task design and general drug effects. A) Participants encoded pictures of outdoor scenes outside the scanner. Picture recognition took place under haloperidol/placebo in the fMRI scanner on the next day. B) Haloperidol effect across all trials on brain activity compared to placebo. Note that increased activity in the right dorsal striatum was also present but its extent ( $k=76$ voxels) and height $(z=3.52)$ did not pass the FWEcorrected threshold of $p<.05$. The inverse contrast (placebo $>$ haloperidol) revealed no significant activity. Activation maps are thresholded at $\mathrm{p}<.05$ (FWE-corrected at cluster-level using a cluster forming threshold at voxel level of $\mathrm{p}<.001)$. HC = high confidence, $M C=$ medium confidence, $L C=$ low confidence.

\section{Recognition session under haloperidol/placebo in the fMRI scanner (T1)}

On the next day, the participants filled out questionnaires assessing their current mood and potential adverse effects of the medication. Blood pressure and pulse were controlled by a physician prior to drug administration. Next, the participants received a tablet containing either $2 \mathrm{mg}$ haloperidol or placebo (based on prior random assignment). In the following waiting period of 2.5 hours, blood pressure and pulse were checked again 30 minutes and 2 hours after drug administration. Also the questionnaires on current mood and adverse medication effects were filled out twice again by the participants. After a brief practice round and familiarization with the recognition task, the participants started the picture recognition inside the scanner approximately 2.5 hours after tablet ingestion (Fig. 1A). During the recognition task, all 80 previously encoded pictures were presented randomly intermixed with 80 new pictures (lures). For each picture (presented for 4 s, ISI 2-4 s), participants indicated the old/new status and their subjective memory confidence on a combined 6-point confidence scale ( 1 - "high confidence old", 6 - "high confidence new") by pressing one of six buttons. The boxes corresponding to the various levels of confidence old/new were horizontally arranged in each trial in random order to minimize transfer-effects to the next rating. Feedback about the correctness of the response was not given. 2-4 seconds after each confidence old/new rating, 
participants indicated how pleasant the preceding memory retrieval was on a visual analogue scale (VAS) ranging from 1 ("not at all") to 100 ("very much"). The pleasantness rating was measured on the continuous VAS (rather than on a categorical scale as the confidence rating) to minimize an implicit transfer from the confidence to the pleasantness ratings. The completion of this task took approximately 45 minutes. After the scanning session, the participants completed another round of the mood and adverse effect questionnaires and their blood pressure and pulse was checked a last time. Another behavioral testing session on the computer followed, including again the working memory tasks (complex span, block span and digit span).

\section{Image acquisition and pre-processing}

Functional MR images were obtained during recognition on a 3T system Siemens Trio using single-shot echo-planar imaging with parallel imaging (GRAPPA (Griswold et al, 2002), in-plane acceleration factor 2) and simultaneous multi-slice acquisitions (Feinberg et al, 2010; Moeller et al, 2010; Setsompop et al, 2012; Xu et al, 2013) ("multiband", slice acceleration factor 2; TR = 1.98s, TE $=26 \mathrm{~ms}$, flip angle $=$ $70^{\circ}, 64$ axial slices, voxel size $2 \times 2 \times 2 \mathrm{~mm}^{3}$ ). The corresponding image reconstruction algorithm was provided by the University of Minnesota Center for Magnetic Resonance Research. In addition, an anatomical high-resolution T1-weighted image $\left(T R=2.3 \mathrm{~s}, T E=2.98 \mathrm{~ms}\right.$, flip angle $=9^{\circ}, 192$ sagittal slices, voxel size $1 \times 1 \times 1 \mathrm{~mm}^{3}$ ) and an anatomical magnetization transfer (MT) image (TR $=14 \mathrm{~ms}, \mathrm{TE}$ $=3.2 \mathrm{~ms}$, flip angle $=6^{\circ}, 240$ coronal slices, voxel size $1 \times 1 \times 1 \mathrm{~mm}^{3}$ ) was acquired for each participant. The data were pre-processed using SPM12 (http://www.fil.ion.ucl.ac.uk/spm/). The first five EPI images were discarded to allow for magnetic-field saturation. EPI images were corrected for motion and for the interaction between motion and distortion (using the unwarping procedure). Anatomical T1-weighted images were normalized to standard MNI space using DARTEL normalization. Subsequently, the EPI images and the MT image were co-registered with the normalized T1 image and the DARTEL normalization parameters were applied to the EPI images and the MT image. Finally, these normalized EPI images were spatially smoothed with a Gaussian kernel of $8 \mathrm{~mm}$ full width at halfmaximum. 


\section{Data analysis}

\section{Behavioral data}

All behavioral data were analyzed using SPSS 21.0.0 (SPSS; Chicago, IL) and Matlab R2013a (The MathWorks; Natick, MA). Post-hoc tests were Bonferroni-corrected where appropriate and corrected p-values are reported.

\section{Side effects and mood}

The scores on the adverse medication effects questionnaire were summed together per measurement time point and analyzed relative to baseline for group differences using a repeated measures ANOVA with the factors group and time. Similarly, pulse and blood pressure measurements were analyzed relative to baseline for group differences using a repeated measures ANOVA with the factors group and time. The 16-item mood questionnaire was analyzed by reversing inverted items and logtransforming all scales before grouping the items into the three dimensions "alertness", "calmness" and "contentment" (Bond and Lader, 1974). The resulting three dimensions were compared between the groups relative to baseline using $2 \times 2$ repeated measures ANOVAs with the factors group and time.

\section{Recognition memory task}

For the recognition performance at $\mathrm{T} 1$, the responses on the 6-point confidence scale were used to compute the accuracy of each trial based on the factual old- or new-status of the item. Trials were sorted post-hoc into the response categories hits (correct old-responses), correct rejections (CR, correct new-responses), false alarms (FA, incorrect old-responses) and misses (incorrect newresponses). Discrimination sensitivity ( $d^{\prime}$ ) and response bias (c) were computed according to the signal detection approach (Macmillan and Creelman, 2004) both across all confidence levels and separately for each confidence level. Discrimination sensitivity was calculated as $d^{\prime}=z(H)-z(F A)$, where $z$ represents the inverse of the cumulative normal distribution and $H=p($ response $=$ old $\mid$ stimulus $=$ old $)$ and $F A=p($ response $=$ old $\mid$ stimulus $=$ new $)$. Response bias was calculated as $c=-0.5[z(H)+z(F)]$. The 
resulting $d^{\prime}$ and $c$ parameters were compared between groups using independent samples t-tests and $d^{\prime}$ and c parameters per confidence level were analyzed with $2 \times 3$ repeated-measures analyses of variance (ANOVAs) with the factors group and confidence level. We moreover analyzed the effects of haloperidol on recollection vs. familiarity processes, which are thought to differentially contribute to memory retrieval (Yonelinas, 2002). According to the dual-process model of recognition memory (Yonelinas et al, 2010), recollection is a threshold process which constitutes the recall of detailed and specific qualitative information. In contrast, familiarity is a quantitative signal detection process, which reflects more global aspects of memory strength. We used the dual process signal detection (DPSD) model as implemented the ROC Toolbox (Koen et al, 2016) to estimate the parameters of recollection and familiarity as well as the receiver operating characteristic (ROC) curves for each participant based on the individual confidence ratings. The resulting recollection and familiarity parameters were averaged and compared between groups using independent-samples t-tests.

Metamemory for old and new picture trials was quantified using the meta-d' framework (Maniscalco and Lau, 2012). Meta-d' aims to quantify metacognitive sensitivity, that is, how well the observer's confidence ratings discriminate between correct and incorrect responses. Meta- $d$ ' can be evaluated with respect to $d^{\prime}$ in order to take into account the observer's discrimination sensitivity by calculating meta- $d^{\prime}-d^{\prime}$ (meta- $d^{\prime}$ difference) as meta- $d^{\prime}$ is expressed in the same units as $d^{\prime}$. Suboptimal metacognition is then reflected by meta-d' values below zero (meta- $d^{\prime}<d^{\prime}$ ) and enhanced metacognition is reflected by meta-d' values above zero (meta-d' $>d^{\prime}$ ) (Maniscalco and Lau, 2012). Response-specific meta-d' values per trial type (old/new) (Maniscalco and Lau, 2014) were calculated using the MATLAB code available at http://www.columbia.edu/ bsm2105/type2sdt, averaged and compared between groups using a $2 \times 2$ repeated-measures ANOVA with the factors group and old/new. For one placebo participant, the modeled meta-d' for old picture trials was more than 4 standard deviations above the mean (z-score $>4.4)$ and thus this participant was excluded from the group analysis. 
Moreover, mean confidence levels (coded from 1 to 3) were computed per response category (hits, $\mathrm{CR}, \mathrm{FA}$, misses) and compared between groups using a $2 \times 2 \times 2$ repeated-measures ANOVA with the factors group, old/new rating and accuracy. Differences in the frequency of low, medium and high confidence responses were analyzed using a $2 \times 2 \times 2 \times 3$ repeated-measures ANOVA with the factors group, old/new rating, accuracy and confidence level to test whether the confidence responses were selected equally often in both groups. Reaction times (RT) differences as well as pleasantness ratings could not be analyzed by the full $2 \times 2 \times 2 \times 3$ model due to the scarceness of trials with high confidence incorrect responses. Therefore, we analyzed RT (based on the median RT per participant) and pleasantness data using an independent t-test to test for group differences and multiple repeatedmeasures ANOVAs to test for all possible group $\times$ condition interactions $(2 \times 2 \times 3$ model collapsed across accuracy with factors group, old/new rating and confidence; $2 \times 2 \times 3$ model collapsed across old/new rating with factors group, accuracy and confidence; $2 \times 2 \times 2$ model collapsed across confidence with factors group, accuracy and old/new rating).

$W M$

Due to fatigue two haloperidol participants did not complete the post-fMRI T1 WM span tasks. Additionally, partial data loss due to technical problems affected three haloperidol and one placebo participant in the T1 WM span tasks. Working memory scores were computed for each of the WM span tests (complex span performance, block span performance forward/backward, digit span performance forward/backward) as well as for the accuracy and the RT of the symmetry rating of the complex span test. The individual To baseline WM measures acquired prior to drug administration were compared between groups using independent samples t-tests. We summarized the five WM span scores into a single T0 and T1 WM span summary score using two different approaches. Firstly, z-scores of each WM span test were computed and averaged into a T0 baseline score and T1 score, respectively. Secondly, for T0 and T1 data separately, a principal components analysis (PCA) was conducted on the individual WM span scores and the resulting first component of T0 and of T1 data was used as a WM span summary score. Group differences were again examined by means of an 
independent t-test (TO baseline WM span summary score) and a repeated measures ANOVA with the factors group and time to test for changes in WM from pre- to post-drug administration. The T0 baseline WM span summary score was moreover used as a covariate in the behavioral and fMRI analysis of memory and confidence effects to control for the potential influence of the individual dopamine baseline on medication response (Cools et al, 2008).

\section{Imaging data}

One placebo dataset was acquired only behaviorally due to scanner problems on that day. For the remaining 53 datasets, univariate single subject (first-level) and group (second-level) statistics were conducted using the general linear model as implemented in SPM12. On the first level, delta functions marking trial-onsets of all corresponding events were convolved with the canonical hemodynamic response function to create an event-related regressor for each condition. Low-frequency signal drifts were removed by employing a highpass filter with a cut-off period of $128 \mathrm{~s}$.

First, in order to test for general group differences throughout the recognition memory task, we set up a first-level model containing a regressor representing all picture onset trials. The continuous variable pleasantness was modeled as a parametric modulator. The resulting individual contrast images (picture onset > implicit baseline, pleasantness) were compared between groups using independent-samples t-tests on the second level.

In a second first-level model, we examined effects of memory by grouping trial onsets into hit trials (correct old-responses), correct rejection trials (CR, correct new-responses), false alarm trials (FA, incorrect old-responses) and miss trials (cf. Behavioral data). For trials with missing responses ( $4 \%$ of all trials in both groups), a nuisance regressor was included in the first-level model. Again, pleasantness was modeled as a parametric modulator. The corresponding four individual contrast images (hits > implicit baseline, CR > implicit baseline, FA > implicit baseline, misses > implicit baseline) of interest were fed into a second-level ANOVA. Retrieval success was evaluated by contrasting hits with CR trials using linear contrasts (Spaniol et al, 2009) and group differences with regard to retrieval 
success were evaluated by computing the group by retrieval success interactions (haloperidol hits > $\mathrm{CR})>$ (placebo hits $>\mathrm{CR}$ ) and (placebo hits $>\mathrm{CR}$ ) $>$ (haloperidol hits $>\mathrm{CR}$ ) on the second level. Additionally, we also set up a second-level model including pleasantness per condition.

For the analysis of confidence and to simultaneously examine confidence effects per response category condition, we used a third first-level model where hit, CR, FA and miss trials were further split into high (HC), medium (MC) and low (LC) confidence trials. As before, we included a nuisance regressor for trials with missing responses and modeled pleasantness as a parametric modulator. The corresponding 12 individual contrast images $(\mathrm{HC} / \mathrm{MC} / \mathrm{LC}$ hits > implicit baseline, HC/MC/LC CR > implicit baseline, HC/MC/LC FA > implicit baseline, $\mathrm{HC} / \mathrm{MC} / \mathrm{LC}$ misses > implicit baseline) of interest were fed into a second-level ANOVA. In the second-level analysis, confidence was evaluated by contrasting $\mathrm{HC}$ with LC trials using linear contrasts and group differences with regard to confidence were evaluated by computing the group by confidence interactions (haloperidol $\mathrm{HC}>\mathrm{LC}$ ) $>$ (placebo $\mathrm{HC}>\mathrm{LC}$ ) and (placebo $\mathrm{HC}>\mathrm{LC}$ ) $>$ (haloperidol $\mathrm{HC}>\mathrm{LC}$ ). We decided to model confidence as a linear contrast rather than as a parametric modulator of trial onsets because of the group differences present for the trial regressors which might pose a problem for the interpretation of parametric regressor differences.

All resulting activation maps were thresholded at $P<.05$ (family-wise error (FWE)-corrected for multiple comparisons). Given the strong a-priori hypothesis of involvement of structures with (presynaptic) dopamine receptors, we used a small volume FWE correction (SVC-FWE) at $P<.05$ based on anatomical masks ( $50 \%$ probability maps) of the striatum and hippocampus created using the Harvard-Oxford cortical and subcortical structural atlases (Desikan et al, 2006) as implemented in FSL (https://fsl.fmrib.ox.ac.uk/fsl/fslwiki/Atlases). For all other reported findings, whole-brain FWEcorrection at cluster level at $P<.05$ (using a cluster-forming height threshold at voxel-level of $P<.001$ (Eklund et al, 2016)) was applied. To localize the dopaminergic midbrain (SN/VTA), we used the mean anatomical magnetization transfer (MT) image. The locus coeruleus (LC) was localized using the probability map (2SD map) of the LC (Keren et al, 2009) available at http://www.eckertlab.org/LC. 
bioRxiv preprint doi: https://doi.org/10.1101/274159; this version posted March 2, 2018. The copyright holder for this preprint (which was not certified by peer review) is the author/funder. All rights reserved. No reuse allowed without permission.

Resulting activations in the hippocampus and the amygdala were anatomically localized with probabilistic maps of cytoarchitectonically defined areas (Amunts et al, 2005) using the SPM Anatomy toolbox (Eickhoff et al, 2005). 


\section{Results}

\section{General drug effects}

Comparison of demographic data and all available test data acquired prior to drug administration demonstrated that the groups did not differ with regard to age, sex, weight or in terms of baseline working memory, general response speed or attention capabilities (Table 1). Moreover, participants were not able to guess the substance received above chance level and there were no group differences in reported side effects or subjective feelings relative to baseline (Table S1), confirming the appropriate blinding of participants. With regard to overall group differences, our fMRI results showed significantly higher activity under haloperidol compared to placebo across all recognition trials specifically and selectively in the dorsal striatum ( $p<.05$ whole-brain FWE-corrected; Fig. 1B). No significant activity differences were found for the inverse contrast (placebo > haloperidol) in any brain region. Importantly, exploration of striatal group differences using an uncorrected threshold did not reveal any striatal voxels showing higher activity for placebo compared to haloperidol.

Table 1: Demographics and baseline performance

\begin{tabular}{|c|c|c|c|}
\hline & Placebo, $n=27$ & Haloperidol , $n=27$ & Statistics \\
\hline Age & 24.37 years \pm 3.3 & 23.56 years \pm 2.5 & $T(52)=1.03, p=.31$ \\
\hline Sex & 8 males, 19 females & 6 males, 21 females & $x^{2}(1)=0.39, p=.54$ \\
\hline Weight & $65.70 \mathrm{~kg} \pm 6.8$ & $65.74 \mathrm{~kg} \pm 10.6$ & $T(52)=-0.15, p=.99$ \\
\hline Baseline WM: Complex & $25.78 \pm 7.3$ & $27.85 \pm 7.0$ & $T(52)=-1.07, p=.29$ \\
\hline span/ symmetry accuracy/ & $96.1 \% \pm 4.2$ & $96.7 \% \pm 3.7$ & $T(52)=-0.51, p=.61$ \\
\hline RT symmetry rating & $2.17 \mathrm{~s} \pm 1.44$ & $1.67 \mathrm{~s} \pm 0.63$ & $\mathrm{~T}(35.49)=1.63, \mathrm{p}=.11$ \\
\hline Baseline WM: digit span & $8.26 \pm 1.3$ & $8.30 \pm 1.7$ & $T(52)=-0.09, p=.93$ \\
\hline forward/ backward & $9.15 \pm 2.2$ & $8.67 \pm 1.7$ & $\mathrm{~T}(52)=0.37, \mathrm{p}=.93$ \\
\hline Baseline WM: block span & $9.73 \pm 1.8$ & $10.44 \pm 2.0$ & $T(51)=-1.35, p=.18$ \\
\hline forward/ backward & $9.15 \pm 1.9$ & $9.81 \pm 1.6$ & $T(51)=-1.37, p=.18$ \\
\hline $\begin{array}{l}\text { Baseline WM: z-summary } \\
\text { score }\end{array}$ & $-0.06 \pm 0.63$ & $0.04 \pm 0.58$ & $T(52)=-0.63, p=.53$ \\
\hline Baseline encoding: & $76.78 \% \pm 22.0$ & $80.23 \% \pm 15.9$ & $T(51)=-0.66, p=.51$ \\
\hline categorization accuracy & $0.58 s \pm 0.04$ & $0.57 s \pm 0.06$ & $\mathrm{~T}(51)=0.71, p=.48$ \\
\hline picture/ RT/ categorization & $86 \% \pm 9.0$ & $86 \% \pm 10.5$ & $\mathrm{~T}(51)=0.12, \mathrm{p}=.91$ \\
\hline accuracy arrows/ RT & $0.38 s \pm 0.01$ & $0.38 \mathrm{~s} \pm 0.02$ & $\mathrm{~T}(51)=0.05, p=.96$ \\
\hline
\end{tabular}

\pm denotes the standard variation 


\section{Differential effects of haloperidol on recognition performance, metamemory and working memory}

Recognition memory performance measured as discrimination sensitivity ( $d^{\prime}$ ) computed according to the signal detection theory (SDT) approach (Macmillan and Creelman, 2004) was significantly enhanced in the haloperidol group $(t(52)=-2.27, p=.028$, Cohen's $d=-0.63 /$ Pearson's $r=0.30$ (medium effect size); Fig. 2B) which was similarly due to more correct responses for old and new items (i.e., more hits and CR; Table 2) but showed no group difference in response bias (Fig. S1C). Correspondingly enhanced recognition memory under haloperidol was observed when characterizing performance with the corrected hit-rate (Fig. S1B). Moreover, analyzing d' per confidence level revealed that the superior discrimination performance under haloperidol was driven by better performance on high confidence $(\mathrm{HC})$ trials (significant group $x$ confidence interaction for $d^{\prime}: F(2,49)$ $=6.20, p=.004$, partial $\eta^{2}=.20$ (large effect size); post-hoc independent samples t-test HC trials: $t(50)$ $=-3.11, p=.009$ (Bonferroni-corrected), Cohen's $d=-0.88 /$ Pearson's $r=0.40$ (large effect size); Fig. 2B). Together, these results indicate that the acute administration of haloperidol boosted retrieval of previously learned information mostly by increasing high confidence correct responses (rather than increasing the amount of correct responses at mid and low confidence range). We additionally analyzed the recognition performance within the framework of the dual processing dual-process signal detection (DPSD) model (Koen et al, 2016). Although there was no group $\mathrm{x}$ memory-type (recollection/familiarity) interaction $(F(1,52) 0.88, p 0.351)$, we analyzed the recollection and familiarity effects separately. This explorative analysis revealed that the haloperidol group had significantly higher estimates of recollection only $(t(52)=-2.29, p=.026$, Cohen's $d=-0.64 /$ Pearson's $r=0.30$ (medium effect size); Fig. 2C); there was no group difference in the estimates of familiarity ( $t$ $(52)=-1.43, p=0.158)$. However, due to the absence of the interaction effect, one should refrain from drawing specific conclusions from this observation. 


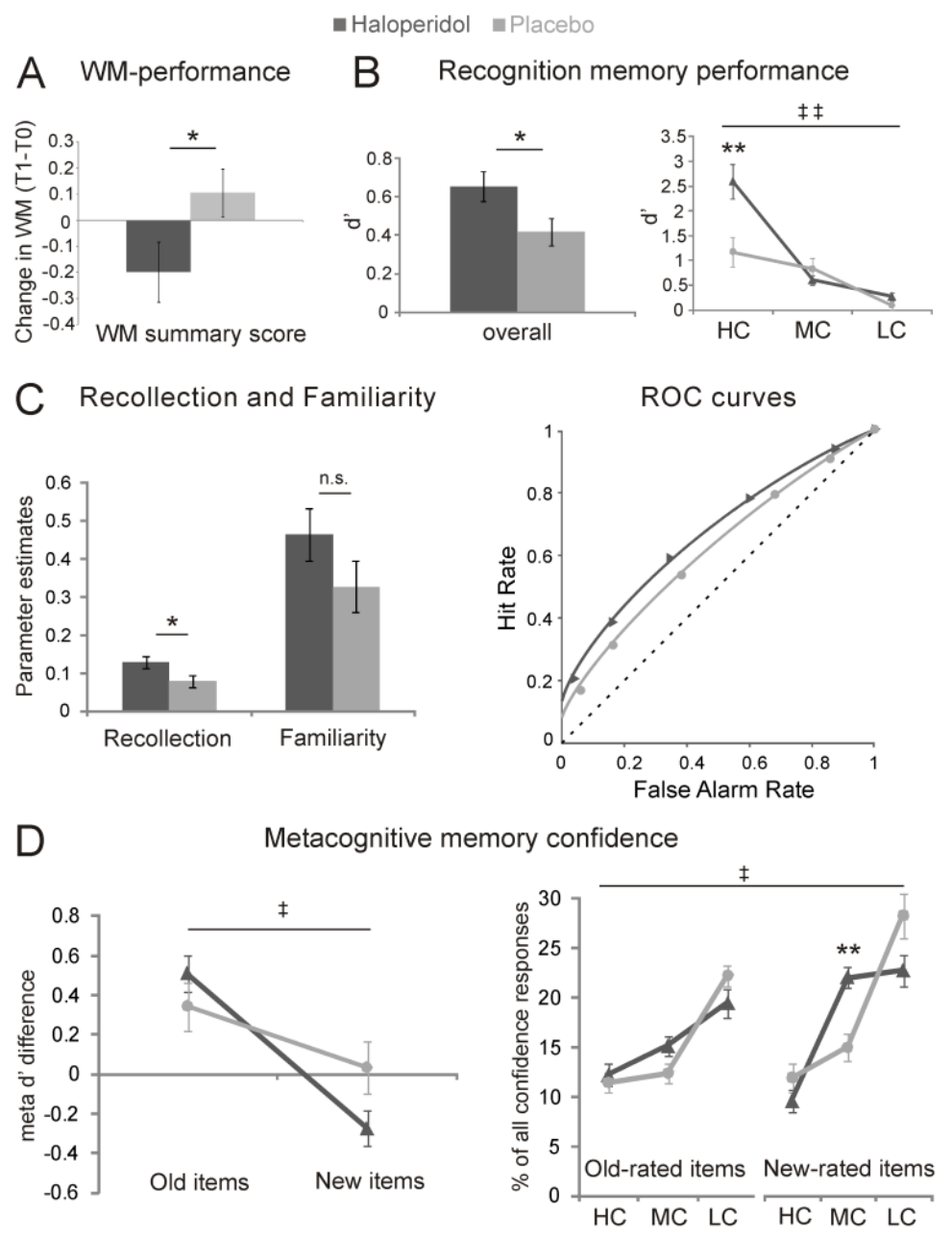

Figure 2: Behavioral effects under haloperidol (dark grey) and placebo (light grey). A) Working memory: Difference in mean z-transformed WM performance after drug administration relative to baseline. B) Memory accuracy: mean d' overall (left) and d' per confidence level (right). C) Mean recollection and familiarity parameters (left) and dual-process model-predicted ROC curves (right) with mean observed ROC values (triangles/circles). D) Metacognition: mean meta d' difference (meta $d^{\prime}-d^{\prime}$ ) for old and new items (left) and frequency of confidence ratings for old and new responses (right). ${ }^{*} / *$ significant group difference at $p<$ $.05 /$ at $p<.01$ (for post-hoc tests after Bonferroni correction); $\neq / \neq \neq=$ significant group $\mathrm{x}$ condition interaction at $\mathrm{p}<.05 /$ at $\mathrm{p}<.01$. n.s. $=$ non-significant. Error bars denote the SEM. $\mathrm{HC}=$ high, $\mathrm{MC}=$ medium, $\mathrm{LC}=\mathrm{low}$ confidence.

In contrast, metacognitive memory confidence showed some impairment under haloperidol. We quantified metamemory for old and new picture items using the meta-d' framework (Maniscalco and Lau, 2012, 2014) which indicates how well the observer's confidence ratings discriminate between correct and incorrect responses. Importantly, meta $d^{\prime}$ is measured in the same units as d' and therefore it can be compared with $d^{\prime}$ to reveal suboptimal (meta- $\left.d^{\prime}<d^{\prime}\right)$ and enhanced $\left(\right.$ meta- $\left.d^{\prime}>d^{\prime}\right)$ 
metacognition by taking into account the observer's discrimination sensitivity (Maniscalco and Lau, 2012). Meta $d^{\prime}$ difference (meta $\left.d^{\prime}-d^{\prime}\right)$ showed a significant group $x$ old $/$ new interaction $(F(1,51)=$ 4.46, $p=.04$, partial $\eta^{2}=.08$ (medium effect size)), demonstrating comparably enhanced metamemory for old items in the haloperidol group as in the placebo group but suboptimal metamemory for new items (Fig. 2D). In a similar vein, the frequency of confidence responses showed a significant shift towards medium confidence (MC) responses particularly for new-rated items, indicating reduced confidence differentiation under haloperidol (group $x$ confidence $x$ old/new rating interaction: $F(2,51)$ $=4.50, p=.016$, partial $\eta^{2}=.15$ (large effect size); post-hoc independent-samples t-test MC new trials: $t(52)=-3.44, p=.006$, Cohen's $d=-0.95 /$ Pearson's $r=0.43$ (large effect size) (Bonferroni-corrected); Fig. 2D). As this shift was due to a relative reduction of both $\mathrm{HC}$ and $\mathrm{LC}$ new-responses, this effect was not reflected by a significant group difference in mean confidence (Table 2; note though that the group $\mathrm{x}$ old/new rating $\mathrm{x}$ accuracy showed some trend towards significance reflecting the higher mean confidence for hits under haloperidol). Together, these analyses point to an impairing effect of acute haloperidol administration on metacognitive confidence for new-decisions.

Table 2: Descriptive statistics of behavioral responses in the recognition memory task.

\begin{tabular}{|c|c|c|c|c|}
\hline Response category & Hits & $\mathrm{CR}$ & FA & Miss \\
\hline Placebo: \% of responses & $53.59(3.1)$ & $61.71(2.7)$ & $38.54(2.9)$ & $46.84(3.3)$ \\
\hline Haloperidol: \% of responses & $59.15(2.0)$ & $65.58(2.5)$ & $35.12(2.6)$ & $40.34(2.0)$ \\
\hline Placebo: mean confidence ${ }^{a}$ & $1.89(0.06)$ & $1.73(0.07)$ & $1.58(0.06)$ & $1.59(0.07)$ \\
\hline Haloperidol: mean confidence ${ }^{a}$ & $1.99(0.05)$ & $1.80(0.04)$ & $1.56(0.05)$ & $1.65(0.05)$ \\
\hline Placebo: mean pleasantness ${ }^{b}$ & $63.34(2.3)$ & $57.66(3.1)$ & $55.33(2.2)$ & $54.15(2.9)$ \\
\hline Haloperidol: mean pleasantness ${ }^{b}$ & $63.74(2.3)$ & $55.79(2.6)$ & $52.76(2.2)$ & $51.51(2.5)$ \\
\hline Placebo: mean $\mathrm{RT}^{\mathrm{c}}$ in $\mathrm{s}$ & $2.12(0.09)$ & $2.17(0.06)$ & $2.15(0.08)$ & $2.16(0.07)$ \\
\hline Haloperidol: mean $\mathrm{RT}^{\mathrm{c}}$ in $\mathrm{s}$ & $1.98(0.08)$ & $2.06(0.08)$ & $2.05(0.08)$ & $2.07(0.08)$ \\
\hline
\end{tabular}

There were no group differences for response times or for pleasantness (Table 2 and Fig. S2B). We additionally repeated all above analyses with the baseline WM span summary score (computed from the five behavioral WM span tasks) included as a covariate to control for individual differences in 
baseline dopamine level (Cools et al, 2008). There was no effect on any of the observed group differences, indicating either that baseline dopamine level did not influence the acute haloperidol effects or that the WM span was not a good proxy for baseline dopamine level. Of interest however, there was an detrimental effect of acute haloperidol on WM measured directly after scanning relative to the baseline WM measured before drug administration: the WM summary score demonstrated decreased WM span under haloperidol (time $x$ group interaction: $F(1,50)=4.28, p=.044$, partial $\eta^{2}=$ .079 (medium effect size); Fig. 2A and Fig. S1A). However, changes in WM scores relative to baseline did not correlate with the meta-d' difference for new-decisions $(r=.03, p=.902)$ or with memory discrimination performance $d^{\prime}(r=.28, p=.172)$ across haloperidol participants. Furthermore, there were no significant correlations of weight with memory discrimination performance $d^{\prime}(r<.01, p=$ $.984)$ or meta-d' difference for new-decisions $(r=.16, p=.434)$ within the haloperidol group. This absence of dosage effects depending on the weight of the participants might not be surprising given the relative restricted range of weight across participants (cf. Table 1).

Increased activity in midbrain and mnemonic regions is linked with better recognition performance Across both groups, the fMRI data revealed a main effect of retrieval success (hits $>C R$ ) in various brain regions including the striatum, PFC, hippocampus, substantia nigra/ventral tegmental area (SN/VTA) and anterior cingulate cortex (ACC) ( $p<.05$ whole-brain FWE-corrected; Fig. 3A). Group differences for retrieval success significant at whole-brain level were evident in an activation cluster localized specifically in the SN/VTA, the hippocampus (presumably CA1 (Amunts et al, 2005)), the basolateral amygdala (Amunts et al, 2005) and the locus coeruleus (LC) under haloperidol compared to placebo (group $x$ retrieval success interaction, whole-brain FWE-corrected at $p<.05$; Fig. $3 \mathrm{~B}$ and Table 3), suggesting that superior memory performance under haloperidol was linked with higher activity in these midbrain and mnemonic regions. Supporting this role for dopaminergic and hippocampal activity in retrieval performance, the activity in the SN/VTA for retrieval success correlated significantly with memory discrimination ( $d^{\prime}$ ) and with right anterior (CA1) hippocampal activity for retrieval success in the haloperidol group (SN/VTA* $d^{\prime}$ : haloperidol group: $r=.47, p=.014$, 
placebo group: $r=.16, p=.445$; SN/VTA*hippocampus: haloperidol group: $r=.50, p=.008$, placebo group: $r=.26, p=.192 ;$ Fig. 3B). As these correlations were not significantly different between groups, these correlation findings merely suggest that participants with higher SN/VTA activity showed better recognition memory and had higher hippocampal activity during retrieval in both groups, although this relationship was more pronounced on a descriptive level in the haloperidol group. Of note, we observed no group differences for this retrieval success activity in the striatum even at an uncorrected threshold despite overall increased striatal activity under haloperidol (see also beta plot in Figure 3A).

Aberrant activity in frontostriatal circuit is linked with impaired metamemory for new-decisions

The main effect of confidence $(\mathrm{HC}>\mathrm{LC})$ across groups revealed similar frontostriatal-parietal regions as previous studies (Clos et al, 2015; Schwarze et al, 2013) (Fig. S2A). The group x confidence interaction showed decreased activity under haloperidol in the PFC and ACC $(p<.05$ whole-brain FWEcorrected) and in the right ventral striatum ( $p<.05$ small volume FWE-corrected; Fig. $3 C$ and Table 3 ). Importantly, the beta parameters in Figure $3 \mathrm{C}$ indicate that this reduced striatal confidence response under haloperidol was due to reduced differentiation between confidence levels rather than due to reduced striatal signal per se: replicating our previous non-pharmacological study (Schwarze et al, 2013), the placebo group showed linear decreasing activity in the striatum with decreasing confidence across response category conditions, but this linear confidence effect was clearly reduced under haloperidol due to an unsystematic activity increase across confidence levels. Note that this effect was particularly evident for new-decisions (CR and miss trials). A similar reduced confidence differentiation but without overall increased activity was observed in the ACC. Including the TO baseline WM span summary score as a covariate in these $\mathrm{fMRI}$ analyses did not change the reported group differences for retrieval success or for confidence. 

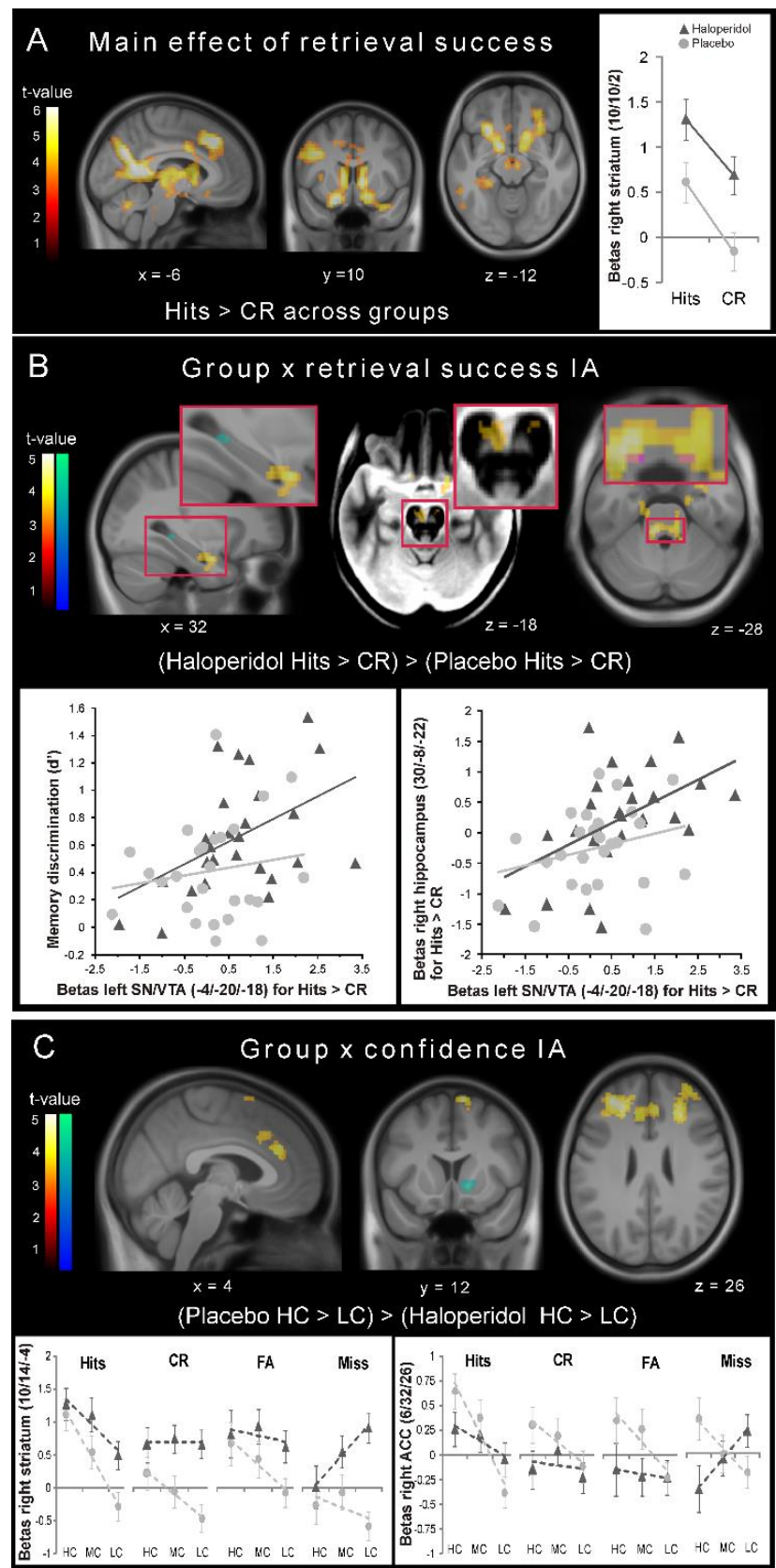

Figure 3: fMRI results. A) Activity pattern of retrieval success across groups and beta estimates from the peak voxel in the right striatum. B) Significantly increased activity for retrieval success under haloperidol in the hippocampus and amygdala (left), SN/VTA (middle; activation displayed on the mean MT image showing the SN/VTA as a bright region) and LC (right; activation overlaid with the LC mask (Keren et al, 2009) in magenta). Scatter plots of the individual SN/VTA response for retrieval success and memory accuracy (left) and the individual anterior hippocampus response for retrieval success (right) in the haloperidol (dark grey triangles) and the placebo group (light grey circles). C) Regions showing higher confidence activity under placebo and beta plots illustrating the confidence pattern in the right striatum and the ACC. Dashed lines represent the linear trend across confidence levels. All activation maps are thresholded at $p<.05$ (warm colors: FWE-corrected at cluster-level using a cluster forming threshold at voxel level of $p<.001$; cold colors: small volume FWE-corrected using anatomical masks). The inverse contrasts revealed no significant activations. $\mathrm{HC}=$ high confidence, $M C=$ medium confidence, $L C=$ low confidence, $I A=$ interaction. 
Table 3: Peak activations group differences

\begin{tabular}{|c|c|c|c|c|c|}
\hline Region & $\mathrm{x}$ & $y$ & $z$ & $z$-score & Cluster size \\
\hline \multicolumn{6}{|l|}{ All trials: $H>P$} \\
\hline L caudate nucleus & -12 & 2 & 18 & 4.74 & 348 \\
\hline \multicolumn{6}{|c|}{ Memory effects: Group x retrieval success $[H$ (Hits $>C R)>P($ Hits $>C R)]$} \\
\hline Brain stem & -8 & -36 & -28 & 4.81 & 420 \\
\hline L LC & -6 & -38 & -30 & 3.75 & \\
\hline R LC & 8 & -38 & -28 & 3.64 & \\
\hline L SN/VTA & -4 & -20 & -18 & 3.41 & \\
\hline R Amygdala (LB(Amunts et al, 2005)) & 30 & 0 & -24 & 4.02 & 189 \\
\hline $\begin{array}{l}R \text { anterior hippocampus (CA1(Amunts et al, } \\
\text { 2005)) }\end{array}$ & 30 & -8 & -22 & 3.68 & \\
\hline $\begin{array}{l}\text { R posterior hippocampus (CA2(Amunts et al, } \\
\text { 2005)) }\end{array}$ & 34 & -34 & -6 & $3.78^{\mathrm{a}}$ & 11 \\
\hline \multicolumn{6}{|c|}{ Confidence effects: Group $x$ confidence $[P(H C>L C)>H(H C>L C)]$} \\
\hline L MFG & -26 & 32 & 24 & 5.27 & 2173 \\
\hline L SFG & -22 & 36 & 26 & 5.22 & \\
\hline R SFG & 16 & 32 & 40 & 4.54 & \\
\hline R ACC & 6 & 32 & 26 & 4.46 & \\
\hline L ACC & -8 & 30 & 26 & 4.33 & \\
\hline R SMA & 10 & 14 & 68 & 4.56 & 230 \\
\hline R NAcc & 10 & 14 & -4 & $4.18^{b}$ & 27 \\
\hline R putamen & 14 & 12 & -6 & $3.93^{b}$ & 23 \\
\hline $\mathrm{R}$ caudate nucleus & 12 & 14 & -2 & $3.91^{b}$ & 36 \\
\hline
\end{tabular}

$\mathrm{x}, \mathrm{y}, \mathrm{z}$ coordinates refer to the peak voxel in MNI space thresholded at $P<0.05$ (FWE-corrected at cluster-level; ${ }^{\text {a) }}$ small volume FWE-corrected using the bilateral anatomical hippocampus mask; ${ }^{b}$ small volume FWE-corrected using the bilateral anatomical striatum mask). No significant activations were observed for the inverse contrasts. R, right; L, left; $H$, haloperidol; $P$, placebo; $L C$, locus coeruleus; SN/VTA, substantia nigra/ ventral tegmental area; MFG, middle frontal gyrus; SFG, superior frontal gyrus; ACC, anterior cingulate gyrus; SMA, supplementary motor area; NAcc, nucleus accumbens.

Together, the results show that the dopaminergic modulation improved hippocampally-mediated recognition performance in the haloperidol group but impaired some aspects of frontostriatallymediated metamemory confidence and working memory. 


\section{Discussion}

We showed here that retrieval of previously learned information in humans is under the influence of dopaminergic signaling and that retrieval of episodic information can be improved by acute low-dose D2 antagonist administration. This enhancement of retrieval performance under the D2 antagonist haloperidol is in agreement with previous reports of haloperidol-induced memory retrieval facilitation in the rat (Chugh et al, 1991; Sara, 1986). In our current study, the superior retrieval discrimination performance in the haloperidol group was associated with higher activity in the SN/VTA, LC, hippocampus and amygdala compared to the placebo group. Striatal activity was overall increased in this recognition task in the haloperidol group, but there was no evidence for striatal contribution to the improved retrieval performance. Instead, striatal and prefrontal effects were seen for metamemory confidence, where reduced confidence differentiation in the striatum and the PFC mimicked the behavioral impairment in metamemory for new decisions.

While chronic and high-dose administration of haloperidol should decrease dopaminergic signaling due to postsynaptic receptor blockade, acute administration of low doses of D2-antagonists is thought to primarily block presynaptic autoreceptors (which have higher affinity for dopamine than the postsynaptic ones (Ford, 2014)) and thereby lead to paradoxical dopamine-stimulating effects (Frank and O'Reilly, 2006a; Knutson and Gibbs, 2007). Indeed, acute administration of D2-antagonists has been shown to potentiate activity of dopamine neurons and dopamine release in the striatum in response to dopamine-evoking stimuli in rodents and non-human primates (Chen et al, 2005; Dugast et al, 1997; Garris et al, 2003; Jaworski et al, 2001; Moghaddam and Bunney, 1990; Pehek, 1999; Pucak and Grace, 1994; Schwerdt et al, 2017; Youngren et al, 1999). Dopamine-stimulating effects are moreover reflected in increased striatal and hippocampal blood flow in rodents (Chen et al, 2005; Schwarz et al, 2004) but also in humans (Handley et al, 2013) in response to acutely administered D2antagonists. Generally, the increased striatal and SN/VTA activations in this recognition task under haloperidol seem hard to reconcile with decreased dopaminergic signaling due to postsynaptic receptor blockade, but rather are in accordance with low-dose haloperidol-induced amplification of 
dopamine release from the SN/VTA. Importantly, optogenetic studies actually demonstrated that phasic stimulation of dopaminergic neurons increases fMRI activity primarily in the dorsal striatum (Ferenczi et al, 2016; Lohani et al, 2016), which corroborates our dopamine-stimulating interpretation of the haloperidol effects in this recognition task.

D2-autoreceptors are particularly abundant in the striatum (Ford, 2014) but also present in the hippocampus (Rocchetti et al, 2015) and amygdala (Bull et al, 1991) and although dopaminestimulating effects in response to D2-antagonists seem to be less extreme in these structures compared to the striatum (Garris and Wightman, 1995), the relative sparseness of DAT in the hippocampus (Kwon et al, 2008) might lead to longer-lasting dopamine effects in the hippocampus (Prince et al, 2016). In particular, such increased stimulation of the postsynaptic D1-like receptors prevailing in hippocampus and amygdala (Köhler et al, 1991; Okubo et al, 1999; Rocchetti et al, 2015) by the dopaminergic midbrain might have led to the improved memory discrimination, possibly mediated by an increased signal-to-noise ratio (Frank, 2005; Warren et al, 2016; Yousif et al, 2016) of the representation of episodic information in these mnemonic structures. For example, dopamine might have affected hippocampal pattern completion (Li et al, 2010; Wilson et al, 2006) or the comparator function of the CA1 region, which compares predicted input computed based on pattern completion in CA3 with actual cortical input during retrieval (Hasselmo et al, 1995). Moreover, the strong LC retrieval success activity observed under haloperidol might point to a possible role of noradrenergic signaling in this recognition task (see also (Sara, 1986)). Previous studies reported that acute haloperidol administration leads to increased LC neuron activity possibly by alpha2-adrenergic receptor blockade (Dinan and Aston-Jones, 1984; Olpe et al, 1983) and noradrenaline released from the LC into the hippocampus and the amygdala is important for retrieval of emotional memories (Thomas, 2015). Finally, the hypothesis of signal-to-noise ratio amplification is even more prominent for norepinephrine than dopamine (Aston-Jones and Cohen, 2005; Warren et al, 2016). However, recent studies revealed that part of the dopaminergic input to the hippocampus actually stems from the dopaminergic co-release of noradrenergic LC neurons (Kempadoo et al, 2016; Smith and Greene, 
2012; Takeuchi et al, 2016). Thus, the strong LC activity for retrieval success might also be due to upregulated dopamine-releasing noradrenergic LC neurons under haloperidol.

In contrast, enhanced dopaminergic stimulation of frontostriatal networks underlying metacognitive (Clos et al, 2015) and cognitive control processes (Scimeca and Badre, 2012) under haloperidol resulted in behavioral impairments in metamemory for new-decisions and probably also contributed to the decreased working memory performance (Frank and O'Reilly, 2006b) in the haloperidol group. In particular, the unsystematically increased striatal activity across confidence levels might have limited the dynamic response range of the striatum (Frank, 2005) to signal different confidence levels and altered frontostriatal interactions with the PFC and ACC. These frontostriatal self-monitoring and cognitive control mechanisms seem to be more important for new-decisions than for old-decisions as only the former were impaired under haloperidol. Note that direct stimulating effects of haloperidol in PFC and ACC are less likely due to the relative rareness of presynaptic D2-autoreceptors in these frontal regions (Bannon et al, 1983; Lammel et al, 2008; Roth, 1984; Wolf and Roth, 1990) and the lack of overall increased activity observed here. However, we can of course not rule out the possibility that the increased striatal activity is caused by reduced inhibition by the PFC under haloperidol rather than the other way around.

In addition to acute haloperidol-induced enhancement of dopamine release, it is possible that postsynaptic effects are present to some degree even at low doses of D2 antagonists. As such, increased dopamine release combined with postsynaptic D2 receptor blockade might decrease the ratio of D2 to D1 receptor activation (Kahnt and Tobler, 2017; Shi et al, 1997). Such an activation shift from D2 to D1 receptors might have additionally contributed to the retrieval performance and metamemory effects reported here, rather than increased dopamine release alone.

Of interest, the striatum still showed differential activity for retrieval success in the haloperidol group to a similar degree as in the placebo group. This absence of a striatal retrieval effect under haloperidol might suggest that previously observed retrieval-related activity in the striatum (Clos et al, 2015) is 
not mediated by dopamine. Possibly, this striatal retrieval activity might reflect a glutamatergic signal of hippocampal origin, which is usually thought to signal novelty (Axmacher et al, 2010; Lisman and Grace, 2005) but possibly also oldness, depending on the goal of the current state (Herweg et al, 2018). However, this null-findings should be treated with some caution as the absence of a striatal retrieval effect might also be due to the lack of power.

Together, these data demonstrate that the dopaminergic modulation here has facilitating effects on midbrain-hippocampal memory retrieval but detrimental effects on frontostriatally-mediated metamemory. These opposing findings are in agreement with recently demonstrated dissociable brain mechanisms underlying recognition performance and memory confidence in non-human primates (Miyamoto et al, 2017) and with dissociative effects of noradrenergic modulation on discrimination accuracy and confidence in sensory decision-making (Allen et al, 2016; Hauser et al, 2017) as well as with long-established behavioral dissociations between memory accuracy and confidence in human cognitive studies (e.g. (Busey et al, 2000; Chandler, 1994; Dobbins et al, 1998; Roediger and DeSoto, 2014)). Moreover, the improved retrieval performance observed here is consistent with previous rat studies reporting memory facilitation after acute systemic haloperidol administration in the retrieval phase (Chugh et al, 1991; Sara, 1986). Finally, our findings might help to explain the presence of episodic memory (Scimeca and Badre, 2012) and metamemory (Eisenacher and Zink, 2017) impairments in dopamine-related diseases as well as offer new perspectives for the treatment of memory disorders.

\section{Limitations}

Our interpretation rests heavily on the assumption that acute administration of $2 \mathrm{mg}$ haloperidol will actually increase dopaminergic signaling. However, this effect has only been directly demonstrated in animals. Previous human fMRI studies using similar doses of haloperidol usually assumed that the drug will decrease dopaminergic signaling (e.g., (Oei et al, 2012; Pleger et al, 2009)), as it does when applied chronically. However, our present fMRI results showing increased activity in striatal and SN/VTA 
regions under haloperidol seem hard to reconcile with a dopamine-decreasing action of acute lowdose haloperidol in this recognition task. Moreover, it should be noted that these previous findings of decreased striatal signal in response to appetitive stimuli under haloperidol often resulted from contrasting rewarding with non-rewarding stimuli. In the light of the reduced striatal differentiation between confidence conditions (but an overall increased striatal activity) observed in the current study, it could be possible that contrasting conditions concealed striatal activity increases under haloperidol in these studies. In other words, reduced striatal differentiation between different conditions might have led to the conclusion of reduced striatal activity, although this activity might effectively have been overall increased (note that contrasting high vs. low confidence in our study resulted in significantly higher activity in the placebo group compared to the haloperidol group). Alternatively, effects of haloperidol might be relatively dependent on the task and the kind of stimuli. Eventually, additional studies including alternative methods such as PET will be necessary to resolve these contradictory drug findings as well to examine the specificity of dopaminergic effects on memory retrieval in more detail. In fact, studies investigating dopaminergic effects on memory retrieval separately from dopaminergic effects on encoding or consolidation are relatively scarce. While two haloperidol studies demonstrated memory facilitation in rats (Chugh et al, 1991; Sara, 1986), other animal studies report no (O'Carroll et al, 2006; Savalli et al, 2015) or, with doses high enough to effectively block postsynaptic D2 receptors, even impairing effects (Blokland et al, 1998) of dopaminergic modulation on retrieval discrimination performance. These contradictory results might indicate that the effects might heavily depend on the dopaminergic drug in question, the dosage, the kind of memory task and the route of administration. In humans, the few previous dopamine antagonist memory studies we are aware of modulated dopamine during both encoding and retrieval (Andreou et al, 2014; Morcom et al, 2010) and therefore are difficult to interpret with regard to retrieval effects specifically.

Moreover, we used a between-group design, which is known to have less power compared to a withingroup design. Although we have a reasonable sample size of 27 participants per group and we show 
bioRxiv preprint doi: https://doi.org/10.1101/274159; this version posted March 2, 2018. The copyright holder for this preprint (which was not certified by peer review) is the author/funder. All rights reserved. No reuse allowed without permission.

strong drug effects both behaviorally and neurally on a whole-brain corrected level, it is possible that some effects were overlooked due to lack of power. In particular the absence of the striatal retrieval effect might be due to too little power and should therefore be interpreted with caution. 
Author contributions: Conceptualization, MC and TS; Methodology, MC and TS; Formal Analysis, MC; Investigation, MC; Writing - Original Draft, MC; Writing - Review \& Editing, MC, NB and TS; Resources - NB and TS; Supervison - TS; Funding Acquisition, NB and TS.

Acknowledgments: This study was supported by a grant from the Deutsche Forschungsgemeinschaft to TS and NB (DFG SO 952/3-1). The funders had no role in study design, data collection and analysis, decision to publish, or preparation of the manuscript. The authors are grateful to the University of Minnesota Center for Magnetic Resonance Research for providing the image reconstruction algorithm for the simultaneous multi-slice acquisitions.

Competing interests: The authors declare no competing financial interests. 


\section{References}

Allen M, Frank D, Schwarzkopf DS, Fardo F, Winston JS, Hauser TU, et al (2016). Unexpected arousal modulates the influence of sensory noise on confidence. Elife 5: .

Amunts K, Kedo O, Kindler M, Pieperhoff P, Mohlberg H, Shah NJ, et al (2005). Cytoarchitectonic mapping of the human amygdala, hippocampal region and entorhinal cortex: intersubject variability and probability maps. Anat Embryol 210: 343-352.

Andreou C, Moritz S, Veith K, Veckenstedt R, Naber D (2014). Dopaminergic modulation of probabilistic reasoning and overconfidence in errors: a double-blind study. Schizophr Bull 40: $558-565$.

Aston-Jones G, Cohen JD (2005). An integrative theory of locus coeruleus-norepinephrine function: adaptive gain and optimal performance. Annu Rev Neurosci 28: 403-450.

Axmacher N, Cohen MX, Fell J, Haupt S, Dümpelmann M, Elger CE, et al (2010). Intracranial EEG correlates of expectancy and memory formation in the human hippocampus and nucleus accumbens. Neuron 65: 541-549.

Bannon MJ, Wolf ME, Roth RH (1983). Pharmacology of dopamine neurons innervating the prefrontal, cingulate and piriform cortices. Eur J Pharmacol 92: 119-125.

Blokland A, Honig W, Prickaerts J (1998). Effects of haloperidol and d-amphetamine on working and reference memory performance in a spatial cone field task. Behav Pharmacol 9: 429-436.

Bond A, Lader M (1974). The use of analogue scales in rating subjective feelings. British Journal of Medical Psychology 47: 211-218.

Bull DR, Bakhtiar R, Sheehan MJ (1991). Characterization of dopamine autoreceptors in the amygdala: a fast cyclic voltammetric study in vitro. Neurosci Lett 134: 41-44.

Busey TA, Tunnicliff J, Loftus GR, Loftus EF (2000). Accounts of the confidence-accuracy relation in recognition memory. Psychon Bull Rev 7: 26-48.

Chandler CC (1994). Studying related pictures can reduce accuracy, but increase confidence, in a modified recognition test. Mem Cognit 22: 273-280. 
Chen Y-Cl, Choi J-K, Andersen SL, Rosen BR, Jenkins BG (2005). Mapping dopamine D2/D3 receptor function using pharmacological magnetic resonance imaging. Psychopharmacology (Berl) 180: 705-715.

Chugh Y, Saha N, Sankaranarayanan A, Sharma PL (1991). Possible mechanism of haloperidolinduced enhancement of memory retrieval. Methods Find Exp Clin Pharmacol 13: 161-164.

Clos M, Schwarze U, Gluth S, Bunzeck N, Sommer T (2015). Goal- and retrieval-dependent activity in the striatum during memory recognition. Neuropsychologia 72: 1-11.

Cole DM, Beckmann CF, Oei NYL, Both S, Gerven JMA van, Rombouts SARB (2013). Differential and distributed effects of dopamine neuromodulations on resting-state network connectivity. Neuroimage 78: 59-67.

Cools R, Gibbs SE, Miyakawa A, Jagust W, D'Esposito M (2008). Working memory capacity predicts dopamine synthesis capacity in the human striatum. J Neurosci 28: 1208-1212.

Desikan RS, Ségonne F, Fischl B, Quinn BT, Dickerson BC, Blacker D, et al (2006). An automated labeling system for subdividing the human cerebral cortex on MRI scans into gyral based regions of interest. Neuroimage 31: 968-980.

Dinan TG, Aston-Jones G (1984). Acute haloperidol increases impulse activity of brain noradrenergic neurons. Brain Res 307: 359-362.

Dobbins IG, Kroll NE, Liu Q (1998). Confidence-accuracy inversions in scene recognition: a remember-know analysis. J Exp Psychol Learn Mem Cogn 24: 1306-1315.

Dugast C, Brun P, Sotty F, Renaud B, Suaud-Chagny MF (1997). On the involvement of a tonic dopamine D2-autoinhibition in the regulation of pulse-to-pulse-evoked dopamine release in the rat striatum in vivo. Naunyn Schmiedebergs Arch Pharmacol 355: 716-719.

Eickhoff SB, Stephan KE, Mohlberg H, Grefkes C, Fink GR, Amunts K, et al (2005). A new SPM toolbox for combining probabilistic cytoarchitectonic maps and functional imaging data. Neuroimage 25: $1325-1335$. 
Eisenacher S, Zink M (2017). The Importance of Metamemory Functioning to the Pathogenesis of Psychosis. Front Psychol 8: 304.

Eklund A, Nichols TE, Knutsson H (2016). Cluster failure: Why fMRI inferences for spatial extent have inflated false-positive rates. Proc Natl Acad Sci USA 113: 7900-7905.

Feinberg DA, Moeller S, Smith SM, Auerbach E, Ramanna S, Gunther M, et al (2010). Multiplexed echo planar imaging for sub-second whole brain FMRI and fast diffusion imaging. PLOS ONE 5: e15710.

Ferenczi EA, Zalocusky KA, Liston C, Grosenick L, Warden MR, Amatya D, et al (2016). Prefrontal cortical regulation of brainwide circuit dynamics and reward-related behavior. Science 351: aac9698.

Ford CP (2014). The role of D2-autoreceptors in regulating dopamine neuron activity and transmission. Neuroscience 282: 13-22.

Frank MJ (2005). Dynamic dopamine modulation in the basal ganglia: a neurocomputational account of cognitive deficits in medicated and nonmedicated Parkinsonism. J Cogn Neurosci 17: 5172.

Frank MJ, O'Reilly RC (2006a). A mechanistic account of striatal dopamine function in human cognition: psychopharmacological studies with cabergoline and haloperidol. Behav Neurosci 120: 497-517.

Frank MJ, O'Reilly RC (2006b). A mechanistic account of striatal dopamine function in human cognition: psychopharmacological studies with cabergoline and haloperidol. Behav Neurosci 120: $497-517$.

Garris PA, Budygin EA, Phillips PEM, Venton BJ, Robinson DL, Bergstrom BP, et al (2003). A role for presynaptic mechanisms in the actions of nomifensine and haloperidol. Neuroscience 118: 819-829.

Garris PA, Wightman RM (1995). Distinct pharmacological regulation of evoked dopamine efflux in the amygdala and striatum of the rat in vivo. Synapse 20: 269-279. 
Griswold MA, Jakob PM, Heidemann RM, Nittka M, Jellus V, Wang J, et al (2002). Generalized autocalibrating partially parallel acquisitions (GRAPPA). Magn Reson Med 47: 1202-1210.

Han S, Huettel SA, Raposo A, Adcock RA, Dobbins IG (2010). Functional significance of striatal responses during episodic decisions: recovery or goal attainment? J Neurosci 30: 4767-4775.

Handley R, Zelaya FO, Reinders AATS, Marques TR, Mehta MA, O'Gorman R, et al (2013). Acute effects of single-dose aripiprazole and haloperidol on resting cerebral blood flow (rCBF) in the human brain. Hum Brain Mapp 34: 272-282.

Hasselmo ME, Schnell E, Barkai E (1995). Dynamics of learning and recall at excitatory recurrent synapses and cholinergic modulation in rat hippocampal region CA3. J Neurosci 15: 52495262.

Hauser TU, Allen M, Purg N, Moutoussis M, Rees G, Dolan RJ (2017). Noradrenaline blockade specifically enhances metacognitive performance. Elife 6: .

Herweg NA, Sommer T, Bunzeck N (2018). Retrieval Demands Adaptively Change Striatal Old/New Signals and Boost Subsequent Long-Term Memory. J Neurosci 38: 745-754.

Jaworski JN, Gonzales RA, Randall PK (2001). Effect of dopamine D2/D3 receptor antagonist sulpiride on amphetamine-induced changes in striatal extracellular dopamine. Eur J Pharmacol 418: 201-206.

Kahnt T, Tobler PN (2017). Dopamine Modulates the Functional Organization of the Orbitofrontal Cortex. J Neurosci 37: 1493-1504.

Kempadoo KA, Mosharov EV, Choi SJ, Sulzer D, Kandel ER (2016). Dopamine release from the locus coeruleus to the dorsal hippocampus promotes spatial learning and memory. Proc Natl Acad Sci USA 113: 14835-14840.

Keren NI, Lozar CT, Harris KC, Morgan PS, Eckert MA (2009). In vivo mapping of the human locus coeruleus. Neuroimage 47: 1261-1267.

Kessels RPC, Berg E van den, Ruis C, Brands AMA (2008). The backward span of the Corsi BlockTapping Task and its association with the WAIS-III Digit Span. Assessment 15: 426-434. 
Kim H (2013). Differential neural activity in the recognition of old versus new events: an activation likelihood estimation meta-analysis. Hum Brain Mapp 34: 814-836.

Knutson B, Gibbs SEB (2007). Linking nucleus accumbens dopamine and blood oxygenation. Psychopharmacology (Berl) 191: 813-822.

Koen JD, Barrett FS, Harlow IM, Yonelinas AP (2016). The ROC Toolbox: A toolbox for analyzing receiver-operating characteristics derived from confidence ratings. Behav Res Methods doi:10.3758/s13428-016-0796-z.

Köhler C, Ericson H, Högberg T, Halldin C, Chan-Palay V (1991). Dopamine D2 receptors in the rat, monkey and the post-mortem human hippocampus. An autoradiographic study using the novel D2-selective ligand 125I-NCQ 298. Neurosci Lett 125: 12-14.

Kwon OB, Paredes D, Gonzalez CM, Neddens J, Hernandez L, Vullhorst D, et al (2008). Neuregulin-1 regulates LTP at CA1 hippocampal synapses through activation of dopamine D4 receptors. Proc Natl Acad Sci USA 105: 15587-15592.

Lammel S, Hetzel A, Häckel O, Jones I, Liss B, Roeper J (2008). Unique properties of mesoprefrontal neurons within a dual mesocorticolimbic dopamine system. Neuron 57: 760-773.

Li F, Wang LP, Shen X, Tsien JZ (2010). Balanced dopamine is critical for pattern completion during associative memory recall. PLOS ONE 5: e15401.

Lisman JE, Grace AA (2005). The hippocampal-VTA loop: controlling the entry of information into long-term memory. Neuron 46: 703-713.

Lohani S, Poplawsky AJ, Kim S-G, Moghaddam B (2016). Unexpected global impact of VTA dopamine neuron activation as measured by opto-fMRI. Mol Psychiatry doi:10.1038/mp.2016.102.

Macmillan NA, Creelman CD (Psychology Press: 2004). Detection Theory: A User's Guide. .

Maniscalco B, Lau H (2012). A signal detection theoretic approach for estimating metacognitive sensitivity from confidence ratings. Conscious Cogn 21: 422-430. 
Maniscalco B, Lau H (2014). Signal Detection Theory Analysis of Type 1 and Type 2 Data: Meta-d', Response-Specific Meta-d', and the Unequal Variance SDT Model. The Cognitive Neuroscience of Metacognition 25-66doi:10.1007/978-3-642-45190-4_3.

Mele A, Avena M, Roullet P, De Leonibus E, Mandillo S, Sargolini F, et al (2004). Nucleus accumbens dopamine receptors in the consolidation of spatial memory. Behav Pharmacol 15: 423-431. Menon M, Jensen J, Vitcu I, Graff-Guerrero A, Crawley A, Smith MA, et al (2007). Temporal difference modeling of the blood-oxygen level dependent response during aversive conditioning in humans: effects of dopaminergic modulation. Biol Psychiatry 62: 765-772.

Miyamoto K, Osada T, Setsuie R, Takeda M, Tamura K, Adachi Y, et al (2017). Causal neural network of metamemory for retrospection in primates. Science 355: 188-193.

Moeller S, Yacoub E, Olman CA, Auerbach E, Strupp J, Harel N, et al (2010). Multiband multislice GEEPI at 7 tesla, with 16 -fold acceleration using partial parallel imaging with application to high spatial and temporal whole-brain fMRI. Magn Reson Med 63: 1144-1153.

Moghaddam B, Bunney BS (1990). Acute effects of typical and atypical antipsychotic drugs on the release of dopamine from prefrontal cortex, nucleus accumbens, and striatum of the rat: an in vivo microdialysis study. J Neurochem 54: 1755-1760.

Morcom AM, Bullmore ET, Huppert FA, Lennox B, Praseedom A, Linnington H, et al (2010). Memory encoding and dopamine in the aging brain: a psychopharmacological neuroimaging study. Cereb Cortex 20: 743-757.

O'Carroll CM, Martin SJ, Sandin J, Frenguelli B, Morris RGM (2006). Dopaminergic modulation of the persistence of one-trial hippocampus-dependent memory. Learn Mem 13: 760-769.

Oei NYL, Rombouts SA, Soeter RP, Gerven JM van, Both S (2012). Dopamine modulates reward system activity during subconscious processing of sexual stimuli. Neuropsychopharmacology 37: 1729-1737. 
Okubo Y, Olsson H, Ito H, Lofti M, Suhara T, Halldin C, et al (1999). PET mapping of extrastriatal D2like dopamine receptors in the human brain using an anatomic standardization technique and [11C]FLB 457. Neuroimage 10: 666-674.

Olpe HR, Jones RS, Steinmann MW (1983). The locus coeruleus: actions of psychoactive drugs. Experientia 39: 242-249.

Peelen MV, Fei-Fei L, Kastner S (2009). Neural mechanisms of rapid natural scene categorization in human visual cortex. Nature 460: 94-97.

Pehek EA (1999). Comparison of effects of haloperidol administration on amphetamine-stimulated dopamine release in the rat medial prefrontal cortex and dorsal striatum. J Pharmacol Exp Ther 289: 14-23.

Pessiglione M, Seymour B, Flandin G, Dolan RJ, Frith CD (2006). Dopamine-dependent prediction errors underpin reward-seeking behaviour in humans. Nature 442: 1042-1045.

Pleger B, Ruff CC, Blankenburg F, Klöppel S, Driver J, Dolan RJ (2009). Influence of dopaminergically mediated reward on somatosensory decision-making. PLoS Biol 7: e1000164.

Prince LY, Bacon TJ, Tigaret CM, Mellor JR (2016). Neuromodulation of the Feedforward Dentate Gyrus-CA3 Microcircuit. Front Synaptic Neurosci 8: 32.

Pucak ML, Grace AA (1994). Evidence that systemically administered dopamine antagonists activate dopamine neuron firing primarily by blockade of somatodendritic autoreceptors. J Pharmacol Exp Ther 271: 1181-1192.

Rocchetti J, Isingrini E, Dal Bo G, Sagheby S, Menegaux A, Tronche F, et al (2015). Presynaptic D2 dopamine receptors control long-term depression expression and memory processes in the temporal hippocampus. Biol Psychiatry 77: 513-525.

Roediger HL, DeSoto KA (2014). Confidence and memory: assessing positive and negative correlations. Memory 22: 76-91.

Rossato JI, Radiske A, Kohler CA, Gonzalez C, Bevilaqua LR, Medina JH, et al (2013). Consolidation of object recognition memory requires simultaneous activation of dopamine D1/D5 receptors 
in the amygdala and medial prefrontal cortex but not in the hippocampus. Neurobiol Learn Mem 106: 66-70.

Roth RH (1984). CNS dopamine autoreceptors: distribution, pharmacology, and function. Ann NY Acad Sci 430: 27-53.

Sara SJ (1986). Haloperidol facilitates memory retrieval in the rat. Psychopharmacology (Berl) 89: 307-310.

Savalli G, Bashir ZI, Warburton EC (2015). Regionally selective requirement for D1/D5 dopaminergic neurotransmission in the medial prefrontal cortex in object-in-place associative recognition memory. Learn Mem 22: 69-73.

Schwarz A, Gozzi A, Reese T, Bertani S, Crestan V, Hagan J, et al (2004). Selective dopamine D(3) receptor antagonist SB-277011-A potentiates phMRI response to acute amphetamine challenge in the rat brain. Synapse 54: 1-10.

Schwarze U, Bingel U, Badre D, Sommer T (2013). Ventral striatal activity correlates with memory confidence for old- and new-responses in a difficult recognition test. PLOS ONE 8: e54324. Schwerdt HN, Shimazu H, Amemori K-I, Amemori S, Tierney PL, Gibson DJ, et al (2017). Long-term dopamine neurochemical monitoring in primates. Proc Natl Acad Sci USA 114: 13260-13265. Scimeca JM, Badre D (2012). Striatal contributions to declarative memory retrieval. Neuron 75: $380-$ 392.

Setsompop K, Gagoski BA, Polimeni JR, Witzel T, Wedeen VJ, Wald LL (2012). Blipped-controlled aliasing in parallel imaging for simultaneous multislice echo planar imaging with reduced gfactor penalty. Magn Reson Med 67: 1210-1224.

Shi WX, Smith PL, Pun CL, Millet B, Bunney BS (1997). D1-D2 interaction in feedback control of midbrain dopamine neurons. J Neurosci 17: 7988-7994.

Smith CC, Greene RW (2012). CNS dopamine transmission mediated by noradrenergic innervation. J Neurosci 32: 6072-6080. 
Spaniol J, Davidson PSR, Kim ASN, Han H, Moscovitch M, Grady CL (2009). Event-related fMRI studies of episodic encoding and retrieval: meta-analyses using activation likelihood estimation. Neuropsychologia 47: 1765-1779.

Takeuchi T, Duszkiewicz AJ, Sonneborn A, Spooner PA, Yamasaki M, Watanabe M, et al (2016). Locus coeruleus and dopaminergic consolidation of everyday memory. Nature 537: 357-362.

Thomas SA (2015). Neuromodulatory signaling in hippocampus-dependent memory retrieval. Hippocampus 25: 415-431.

Unsworth N, Redick TS, Heitz RP, Broadway JM, Engle RW (2009). Complex working memory span tasks and higher-order cognition: a latent-variable analysis of the relationship between processing and storage. Memory 17: 635-654.

Warren CM, Eldar E, Brink RL van den, Tona K-D, Wee NJ van der, Giltay EJ, et al (2016). Catecholamine-Mediated Increases in Gain Enhance the Precision of Cortical Representations. J Neurosci 36: 5699-5708.

Wilson IA, Gallagher M, Eichenbaum H, Tanila H (2006). Neurocognitive aging: prior memories hinder new hippocampal encoding. Trends Neurosci 29: 662-670.

Wolf ME, Roth RH (1990). Autoreceptor regulation of dopamine synthesis. Ann N Y Acad Sci 604: $323-343$.

Wrobel N, Wiech K, Forkmann K, Ritter C, Bingel U (2014). Haloperidol blocks dorsal striatum activity but not analgesia in a placebo paradigm. Cortex 57: 60-73.

Xu J, Moeller S, Auerbach EJ, Strupp J, Smith SM, Feinberg DA, et al (2013). Evaluation of slice accelerations using multiband echo planar imaging at 3 T. Neuroimage 83: 991-1001. Yonelinas AP (2002). The nature of recollection and familiarity: A review of 30 years of research. Journal of Memory and Language 46: 441-517.

Yonelinas AP, Aly M, Wang W-C, Koen JD (2010). Recollection and familiarity: examining controversial assumptions and new directions. Hippocampus 20: 1178-1194. 
Youngren KD, Inglis FM, Pivirotto PJ, Jedema HP, Bradberry CW, Goldman-Rakic PS, et al (1999).

Clozapine preferentially increases dopamine release in the rhesus monkey prefrontal cortex compared with the caudate nucleus. Neuropsychopharmacology 20: 403-412.

Yousif N, Fu RZ, Abou-El-Ela Bourquin B, Bhrugubanda V, Schultz SR, Seemungal BM (2016).

Dopamine Activation Preserves Visual Motion Perception Despite Noise Interference of Human V5/MT. J Neurosci 36: 9303-9312. 